INPLASY

PROTOCOL

To cite: Zheng et al.

Comparison of clinical features and laboratory parameters in

children with complete and incomplete Kawasaki disease: a Meta-analysis. Inplasy protocol 202150070. doi: 10.37766/inplasy2021.5.0070

Received: 17 May 2021

Published: 18 May 2021

Corresponding author:

Tai Zheng

609368113@qq.com

Author Affiliation:

Department of Pediatrics, The

Central Hospital of Jiangjin.

Support: No financial support.

Review Stage at time of this submission: The review has not yet started.

Conflicts of interest:

None declared.

\section{Comparison of clinical features and laboratory parameters in children with complete and incomplete Kawasaki disease: a Meta-analysis}

\author{
Zheng, T1; Dai, Q2; Zhang, $\mathrm{H}^{3}$.
}

Review question / Objective: Kawasaki disease is a systemic vascular disease that tends to involve the coronary arteries and is common in pediatric patients. However, some patients have insignificant clinical features and belong to incomplete Kawasaki disease. Due to the atypical clinical features of incomplete Kawasaki disease (IKD), an accurate diagnosis cannot be made early. Various related studies have also reported differences in clinical characteristics between complete and incomplete Kawasaki disease, but no consensus opinion has been formed. Therefore, this study aimed to systematically evaluate the differences in clinical features and diagnostic parameters between complete and incomplete Kawasaki disease, expecting to provide a basis for differentiating complete and incomplete Kawasaki disease.

Condition being studied: Kawasaki disease. The author has consulted relevant literatures, and no similar Meta analysis has been found. Moreover, the number of relevant original literatures is sufficient, so that it can be used for Meta analysis.

INPLASY registration number: This protocol was registered with the International Platform of Registered Systematic Review and Meta-Analysis Protocols (INPLASY) on 18 May 2021 and was last updated on 18 May 2021 (registration number INPLASY202150070).

\section{INTRODUCTION}

Review question / Objective: Kawasaki disease is a systemic vascular disease that tends to involve the coronary arteries and is common in pediatric patients. However, some patients have insignificant clinical features and belong to incomplete Kawasaki disease. Due to the atypical clinical features of incomplete Kawasaki disease (IKD), an accurate diagnosis cannot be made early. Various related studies have also reported differences in clinical characteristics between complete and incomplete Kawasaki disease, but no consensus opinion has been formed. Therefore, this study aimed to 
systematically evaluate the differences in clinical features and diagnostic parameters between complete and incomplete Kawasaki disease, expecting to provide a basis for differentiating complete and incomplete Kawasaki disease.

Condition being studied: Kawasaki disease. The author has consulted relevant literatures, and no similar Meta analysis has been found. Moreover, the number of relevant original literatures is sufficient, so that it can be used for Meta analysis.

\section{METHODS}

Participant or population: Children with complete and incomplete Kawasaki disease.

Intervention: Incomplete Kawasaki disease.

\section{Comparator: Complete Kawasaki disease}

Study designs to be included: Cohort study.

Eligibility criteria: Inclusion criteria (1) The types of studies selected for this metaanalysis were cohort studies; (2) The experimental group of the study was IKD patients; the control group was CKD patients; (3) The included studies needed to contain at least one of the following indicators: Extremity changes, Rash polymorphic, Contival Hyperemia/ Conjunctivitis, Lip/oral mucosal changes, Cervical lymph nodes, platelet count (PLT), white blood cell count (WBC), hemoglobin $(\mathrm{Hb})$, erythrocyte sedimentation rate (ESR) and C-reactive protein (CRP); (4) The observed subjects of the included studies were KD patients with definite diagnosis; (5) The reported data in the literature were complete. Exclusion criteria : (1) The results are not completely statistically analyzed or relevant data are insufficient; (2) Repeated publication of literature; (3) The study subjects are not CKD and IKD; (4) Conference, meta-analysis, and review literature.

Information sources: PubMed, Embase, Cochrane Library, CNKI, VIP, Wangfang Database, and CBM.
Main outcome(s): Extremity changes, Rash polymorphic, Conjunctival Hyperemia/ Conjunctivitis, Lip/oral mucosal changes, Cervical lymph nodes, platelet count (PLT), white blood cell count (WBC), hemoglobin $(\mathrm{Hb})$, erythrocyte sedimentation rate (ESR) and C-reactive protein (CRP).

Quality assessment / Risk of bias analysis: The included studies were cohort studies. Quality assessment of cohort studies was performed using the Newcastle-Ottawa (NOS) scale, a quality evaluation tool specifically for case-control studies and cohort studies. The evaluation included three aspects: selection (four items), comparability (one item), and outcome (three items). Among them, the maximum score of each item of selection and outcome was 1 , the full score of comparable items was 2 , and the total score of scale evaluation results was 9 . Scores $(0 \sim 4)$ were classified as low-quality articles and $(5 \sim 9)$ as high-quality articles.

Strategy of data synthesis: The retrieved literature was initially screened by two investigators independently according to the inclusion and exclusion criteria and then cross-checked. The controversial literature was evaluated by the third party and then unified by discussion.

Subgroup analysis: Sensitivity analyses were performed by country, publication year, child age, etc.

Sensitivity analysis: Sensitivity analyses were performed on outcome measures with high heterogeneity.

Country(ies) involved: China.

Keywords: Kawasaki disease; coronary artery; incomplete Kawasaki disease; Meta-analysis.

Contributions of each author:

Author 1 - Tai Zheng.

Author 2 - Qinyun Dai.

Author 3 - Hui Zhang. 